\title{
Level Adiabatic Model for Dissociation of Diatomic Molecules
}

\author{
G. Smekhov, A. Sergievskaya, M. Pogosbekian, A. Kroupnov \\ Institute of Mechanics, Lomonosov Moscow State University,, \\ Moscow, 119192, Russia \\ sergievskaya@imec.msu.ru,pogosbekian@imec.msu.ru
}

\begin{abstract}
In strong shock waves in rarefied gases chemical processes occur in conditions of thermodynamic nonequilibrium. The big interest is the creation of level models of processes for obtain a rate coefficient. The paper presents a new level model for dissociation, obtained within the adiabatic approximation. Model was verified on the basis of available data of quasiclassical trajectory calculations, which analyzed the dissociation of $\mathrm{N}_{2}$ molecules in a wide temperature range. Comparison of the model with the Marrone - Treanor model and with existing modifications of the Marrone - Treanor models were discussed.
\end{abstract}

Keywords: thermally non-equilibrium conditions, level approximation, dissociation, quasclassical trajectory calculations, rate coefficient.

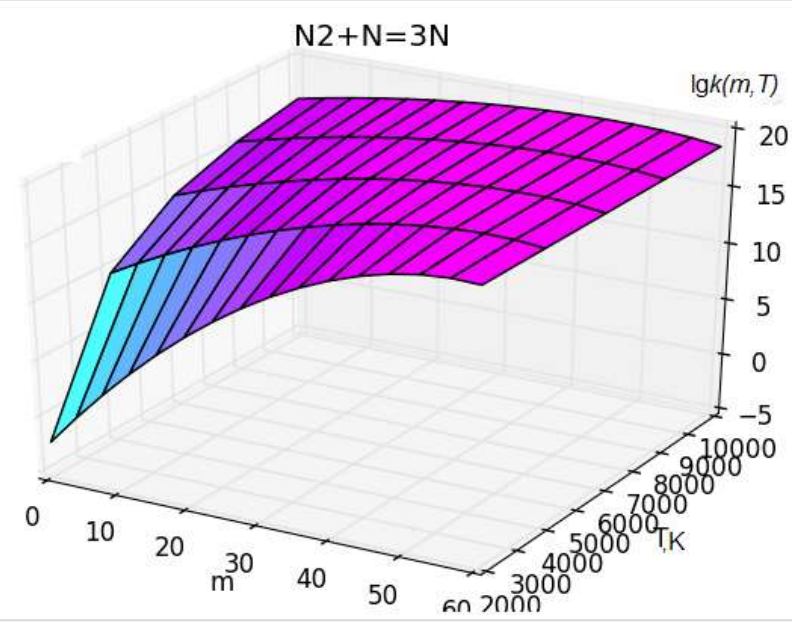

Dependence rate coefficient of dissociation $\mathrm{N}_{2}+\mathrm{N} \rightarrow \mathrm{N}+\mathrm{N}+\mathrm{N}$ on vibrational level and translational temperature $T$ 


\title{
Уровневая адиабатическая модель диссоциации двухатомных молекул
}

\author{
Г. Д. Смехов, А. Л. Сергиевская, М. Ю. Погосбекян, А. А. Крупнов \\ НИИ Механики Московского государственного университета им. М.В. Ломоносова, \\ Россия, Москва, 119192, Мичуринский проспект, 1 \\ sergievska@imec.msu.ru, pogosbekian@imec.msu.ru
}

\begin{abstract}
Аннотация
В условия термодинамической неравновесности происходят различные химические процессы, в числе которых реакции диссоциации занимают важное место и для описания которых, необходимо знание их различных характеристик. Наибольший интерес вызывает разработка уровневых моделей процессов для получения констант скорости. В работе представлена новая уровневая адиабатическая модель диссоциации, полученная в пределах адиабатического приближения. Проведена верификация модели на имеющихся данных квазиклассических траекторных расчетов диссоциации молекул $\mathrm{N}_{2}$ в широком температурном диапазоне, дано сравнение этой модели с моделями Мэрроуна-Тринора и существующими модификациями модели Мэрроуна - Тринора
\end{abstract}

Ключевые слова: термически неравновесные условия, уровневое приближение, диссоциация, квазиклассические траекторные расчеты, константа скорости.

\section{1. Введение}

Исследование характеристик и свойств физико-химических процессов в газе в отсутствие равновесия между колебательными и поступательными степенями свободы требуется для решения современных задач науки и техники, в частности, для развития лазеров различного назначения, для описания космических исследований, сверхзвуковых и гиперзвуковых полетов и т.п.

Исследованию такой характеристики, как константа скорости диссоциации посвящено большое число работ как экспериментальных, так и теоретических работ (см. обзор литературы в [1] или в [2]). В случае слабых отклонений от термического равновесия для представления константы скорости обычно применяется закон Аррениуса, параметры которого определяются экспериментально, эмпирически и теоретически и собраны в существующих обзорах или базах данных [3-6]. В условиях сильного колебательного возбуждения закон Аррениуса, основанный на равновесном больцмановском распределении молекул по колебательным уровням, не применим.

Для расчета скорости реакции в таких условиях использовались в двухтемпературном приближении разнообразные модели, а в уровневом представлении для диссоциации - две эмпирические модели (модели Лосева и Левицкого) и модель Мэрроуна-Тринора, структурированное описание которых можно найти в Каталоге моделей физико-химических процессов [7]. Модель Лосева и Мэрроуна-Тринора получили широкое распространение в силу своей простоты, тем не менее, их точность далеко не всегда удовлетворительна. Эти модели имеют свои, достаточно весомые, ограничения. Модель Лосева демонстрирует простую зависимость уровневой константы скорости от температуры и годится скорее для оценок величины этой характеристики. Эмпирический параметр модели предложен автором для диссоциации только 
двух молекул - $\mathrm{N}_{2}$ и $\mathrm{O}_{2}$ и только для коллайдеров из системы атомов C-N-O. А в модели Мэрроуна-Тринора существенную роль играет эмпирический параметр, значение которого дается автором из общих соображений. Однако, этот параметр, как, впрочем, и параметр модели Лосева, может быть настроен для конкретной реакции при наличии у исследователя более точных расчетных данных, полученных методом молекулярной динамики (МКТ).

В печати все чаще появляются результаты траекторных расчетов характеристик термически неравновесных процессов [8-15].

Моделирование траектории сталкивающихся и реагирующих частиц становится менее уникальной процедурой, что позволяет исследователю рассчитывать константы скорости реакции и вероятности для различных условий и использовать МКТ-результаты как в DSMC, так и в CFD расчетах. Однако, метод МКТ не идеален. Действительно, неопределенности или ошибки, присущие МКТ, включают неопределенности расчета электронной структуры $a b$ initio, погрешности установки PES, статистические ошибки МКТ расчета и т. д. Количественная оценка всех таких неопределенностей является трудной задачей. Кроме того, для многих молекулярных систем не существует ab initio PES. Чувствительность результатов к деталям PES подробно не изучалась. Кроме того, большинство доступные ab initio PES были вычислены только для одного электронного состояния и влияние электронных неадиабатических переходов еще неизвестно.

Помимо эмпирических моделей и приближений на основе МКТ, существует третий подход - традиционный: теоретические (физико-математические) модели процессов, основанные на разумных теоретических положениях, предположениях и приближениях. Аналитические формулы, основанные на фундаментальных физических законах, могут быть получены и использованы в прикладных задачах. Эти модели также не ограничены наличием ab initio PES.

Целью данной работы является представление новой теоретической уровневой физикоматематической модели процесса диссоциации, полученной в пределах адиабатического приближения, определение пределов применимости этой модели и ее верификация на имеющихся данных МКТ расчетов диссоциации молекул $\mathrm{N}_{2}$ в широком температурном диапазоне, а также сравнение с имеющимися уровневыми моделями или их модификациями.

Содержание этой статьи организовано следующим образом. Основные предположения и описание уровневой модели диссоциации представлены в первой части. Затем идет анализ свойств модели на примерах реакций диссоциации молекул $\mathrm{N}_{2}$, сравнение с результатами, полученными с помощью уровневых моделей Мэрроуна-Тринора и Савельева [16], и результатами МКТ расчетов по диссоциации молекул $\mathrm{N}_{2}$, выполненных в Институте механики МГУ [17].

\section{2. Уровневая адиабатическая модель}

Адиабатическая модель диссоциации в модовом (двухтемпературном) приближении была сформулирована Г.Д. Смеховым достаточно давно [18-21], использовалась при верификации моделей диссоциации [22,23], в прикладных проектах, включена в цитируемые выше Справочник [1] и Каталог [7]. Модель основана на предположении о том, молекула в любом колебательном состоянии, включая и основное состояние, может подвергнуться процессу распада в результате столкновения с некоторой частицей М. Процесс диссоциации носит пороговый характер. Энергетический порог диссоциации учитывает снижение энергии диссоциации за счет колебательно-вращательного возбуждения.

Величина сечения диссоциации определяется адиабатическим принципом, так что

$$
\sigma=\sigma_{0} \exp (-\xi)
$$

где $\sigma_{0}$-эффективное сечение распада молекулы в состояниях, находящихся вблизи предела диссоциации и для которых параметр Месси $\xi \ll 1$;

$\xi=k \Delta E_{m} / h \alpha_{0}-$ адиабатический параметр Месси; 
$\Delta E_{m}=D_{0}-E_{m}-$ дефект энергии между диссоциированным и колебательно-возбужденным состоянием молекулы;

$E_{m}$ - энергия $m$-го колебательного уровня;

$D_{0}$ - энергия диссоциации молекулы, выраженные в единицах температуры;

$\alpha_{0}$ - параметр межмолекулярного взаимодействия сталкивающихся частиц;

$h$ - постоянная Планка.

Эффективное сечение диссоциации с $m$-го колебательного уровня вблизи порога диссоциации аппроксимируется ступенчатой функцией. Величина сечения определяется адиабатическим принципом Месси. Поэтому значение $\sigma_{0}$ можно считать близким к газокинетическому сечению столкновения. Распределение молекул по вращательным и колебательным уровням является больцмановским; вращательная температура $T_{r}$ равна поступательной температуре газа $T$ и колебательной температуре $T_{V}$.

На этих же предположениях строится и предлагаемая в данной работе уровневая адиабатическая модель. Ниже дается структурированное описание двух новых целевых функций этой модели - уровневой константы скорости и относительного уровневого фактора, используемое при включении представляемой модели процесса диссоциации в Каталог моделей. При этом константа скорости реакции в уровневом описании представляется в принятом в Каталоге виде

$$
k(m, T)=k^{\prime}(T) g(m, T),
$$

где $k^{\prime}(T)$ - нормировочный множитель, а $g(m, T)$ - абсолютный уровневый фактор.

А относительный уровневый фактор принимается в виде

$$
G\left(m, m^{*}, T\right)=\frac{g(m, T)}{g\left(m^{*}, T\right)},
$$

где $m^{*}$ - фиксированный при исследовании уровень.

Модель была включена в Каталог моделей как 4.8.8 Уровневая адиабатическая модель диссоциации (уровень CVL) с возможностью расчета двух целевых функций: «ЦФ 94. Уровневая константа скорости диссоциации двухатомных молекул» и как «ЦФ 95. Относительный уровневый фактор».

На рис. 1 представлен образ реализация ЦФ 94 в среде Каталога.

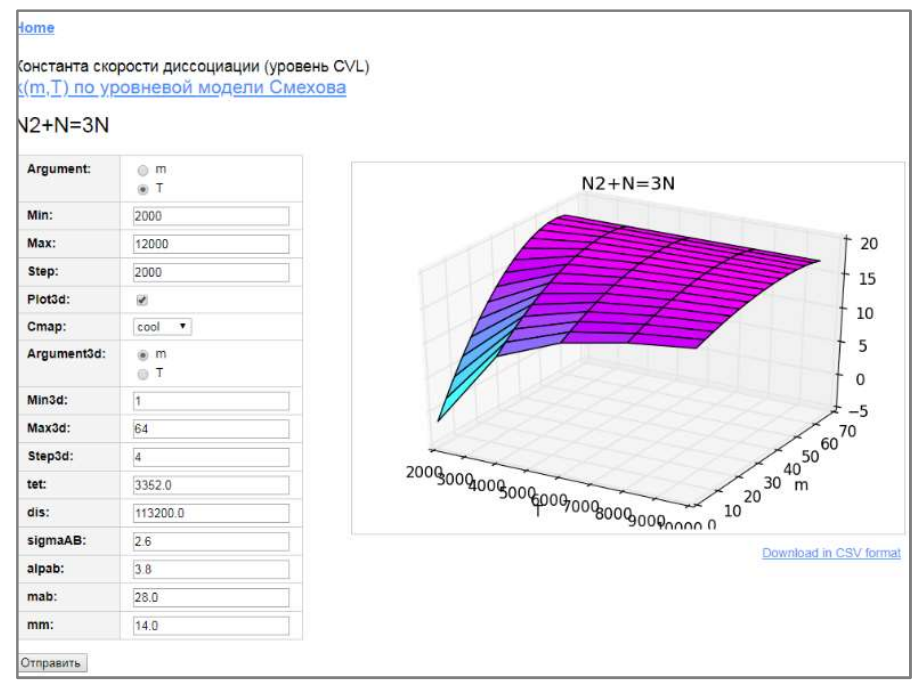

Рис. 1. Расчет уровневой константы скорости диссоциации молекулы $\mathrm{N}_{2}$ в среде Каталога моделей 


\section{Уровневая адиабатическая модель диссоциации двухатомных молекул$$
\mathbf{A B}(\mathbf{m})+\{\mathbf{M}\} \rightarrow \mathbf{A}+\mathbf{B}+\{\mathbf{M}\}
$$

\section{Назначение модели}

Определение уровневой константы скорости и относительного уровневого фактора реакции диссоциации.

\section{Допущения}

1. Диссоциирующая молекула моделируется ангармоническим осциллятором Морзе и жестким ротатором. Распределение молекул по вращательным и колебательным уровням является больцмановским; вращательная температура $T_{r}$ равна поступательной температуре газа $T$.

2. Энергетический порог диссоциации учитывает снижение энергии диссоциации за счет колебательно-вращательного возбуждения.

3. Эффективное сечение диссоциации с $m$-го колебательного уровня вблизи порога диссоциации аппроксимируется ступенчатой функцией. Величина сечения определяется адиабатическим принципом Месси.

\section{Ограничение}

Модель применима при температуре $T \leq D_{0} / 2$.

\section{ЦФ 94. Уровневая константа скорости диссоциции $k(m, T)$}

- $[k]=\mathrm{cm}^{3} /$ моль $\mathbf{c}$;

$$
[\mathrm{AB}(m)+\{\mathrm{M}\} \rightarrow \mathrm{A}+\mathrm{B}+\{\mathrm{M}\}]
$$

- аргумент целевой функции: $T$;

- параметр целевой функции: $m$;

- ограничения:

1. аргумента: $300 \leq T \leq D_{0} / 2$;

2. параметра: $0 \leq m \leq m_{\max }^{h}, m_{\max }^{h}=\left[D_{0} / \theta\right]$;

- замечания:

$\mathrm{AB}(m)$ - ангармонический осциллятор

$D_{0}$ - энергия диссоциации двухатомной молекулы $\mathrm{AB}$

\section{Расчетные формулы}

$$
\begin{aligned}
& k(m, T)=\left(\frac{8 \pi k T}{\mu}\right)^{1 / 2} \sigma_{0} \exp \left(-\beta \sqrt{\Delta E_{m}}\right) \exp \left(-\frac{\Delta E_{m}}{T}\right)\left(-\frac{\Delta E_{m}}{T}\right)\left(1+\frac{\Delta E_{m}}{2 T}\right) \\
& \Delta E_{m}=D_{0}-E_{m}, \quad m=0,1,2, \ldots, m_{\max }, \quad m_{\max }=2\left[\frac{D_{0}}{\theta}\right]-\text { целая часть } \\
& \sigma_{0}=\pi \sigma^{2}, \quad \beta=\frac{1}{h} \sqrt{\frac{k}{2 N_{A}}} \frac{\sqrt{\mu}}{\alpha}, \quad \alpha=\alpha^{\mathrm{AB}}=\frac{1}{2}\left(\alpha^{\mathrm{AA}}+\alpha^{\mathrm{BB}}\right),
\end{aligned}
$$

где

$\sigma \quad$ - параметр потенциала Леннарда - Джонса для потенциала взаимодействия частиц А и В;

$\alpha$ - обратный радиус для пары частиц А и В, вычисляемые согласно по правилу комбинирования;

$\alpha^{\mathrm{AA}}, \alpha^{\mathrm{BB}}$ - обратные радиусы столкновений соответственно частиц АА, ВВ;

$\mu \quad$ - приведенная масса сталкивающихся частиц;

$m_{\mathrm{AB}}, m_{\mathrm{M}}-$ массы молекулы АВ и частицы М;

$E_{m} \quad$ - энергия $m$-го колебательного уровня молекулы, выраженная в градусах; 
$m$ - число колебательных уровней ангармонического осциллятора Морзе;

$N_{\mathrm{A}}$ - число Авогадро;

$k \quad-$ постоянная Больцмана;

$h$ - постоянная Планка;

$D_{0}, E_{m}$ - выражаются в Кельвинах.

\section{Характерные величины и примеры}

Для примера в статье приведены результаты применения уровневой адиабатической модели к реакциям диссоциации азота $\mathrm{N}_{2}+\mathrm{N} \rightarrow \mathrm{N}+\mathrm{N}+\mathrm{N}$ и кислорода $\mathrm{O}_{2}+\mathrm{O} \rightarrow \mathrm{O}+\mathrm{O}+\mathrm{O}$.

Ниже представлены результаты применения ЦФ 94 в двух конструктивных формах: по аргументу $m$ с параметрическим заданием газовой температуры $\{T\}$ и по аргументу $T$ с параметрическим заданием колебательного уровня $m$, то есть данные по $k(m,\{T\})$ и $k(\{m\}, T)$ соответственно. Согласно ограничению, указанному автором, модель применима при температурах $T \leq D_{0} / 2$, т.е. в случае диссоциации $\mathrm{N}_{2}$ это означает, что $T<56600 \mathrm{~K}$ и в случае диссоциации $\mathrm{O}_{2}-T<29680 \mathrm{~K}$, что в обоих случаях значительно превышает реально исследуемые температуры.

Ограничение на максимальное значение колебательных уровней, используемое в модели, выражаются соответственно для $\mathrm{N}_{2} m_{\max }=67$ и для $\mathrm{O}_{2} m_{\max }=52$.

Для реакции диссоциации молекулы $\mathrm{N}_{2}$ при столкновении с атомом $\mathrm{N}$ был выбран температурный диапазон $T=2000 \div 10000$ с параметрическим списком $\{m\}=0,10,20,30,40$ и диапазон номеров колебательных уровней $m=0 \div 60$ с параметрическим списком $\{T\}=3000$, $6000,9000 \mathrm{~K}$. Построены соответствующие семейства: $\lg k(\{m\}, T)$ - на рис. 2 и $\lg k(m,\{T\})$ - на рис. 3 соответственно (константа скорости этой реакции по двум конструктивным формам).

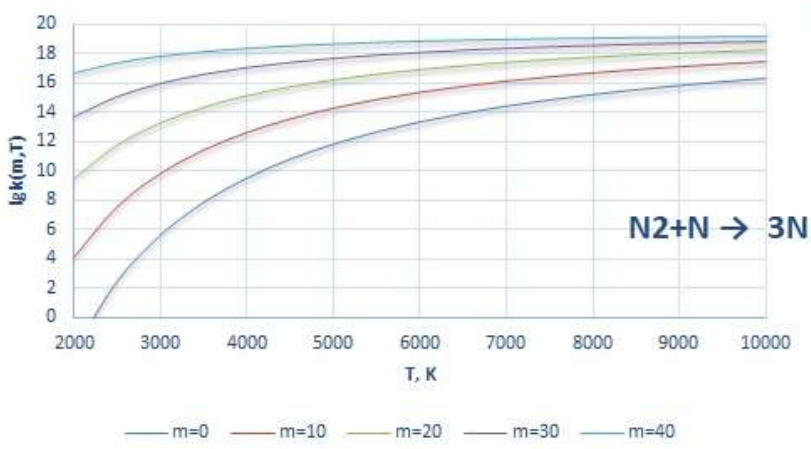

Рис. 2. Уровневая константа скорости диссоциации азота в зависимости от поступательной температуры

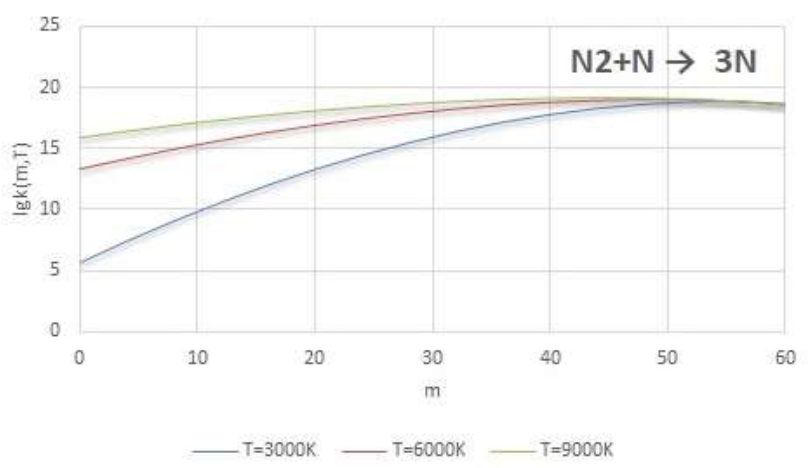

Рис. 3. Уровневая константа скорости диссоциации азота в зависимости от колебательного уровня

Для реакции диссоциации молекулы $\mathrm{O}_{2}$ при столкновении с атомом О был выбран температурный диапазон $T=1000 \div 10000$ с параметрическим списком $\{m\}=0,10,20,30,40$ и диапазон номеров колебательных уровней $m=0 \div 52$ с параметрическим списком $\{T\}=1000$, $4000,8000 \mathrm{~K}$. Построены соответствующие семейства: на рис. $4-\lg k(\{m\}, T)$ и $\lg k(m,\{T\})$ на рис. 5 соответственно. 


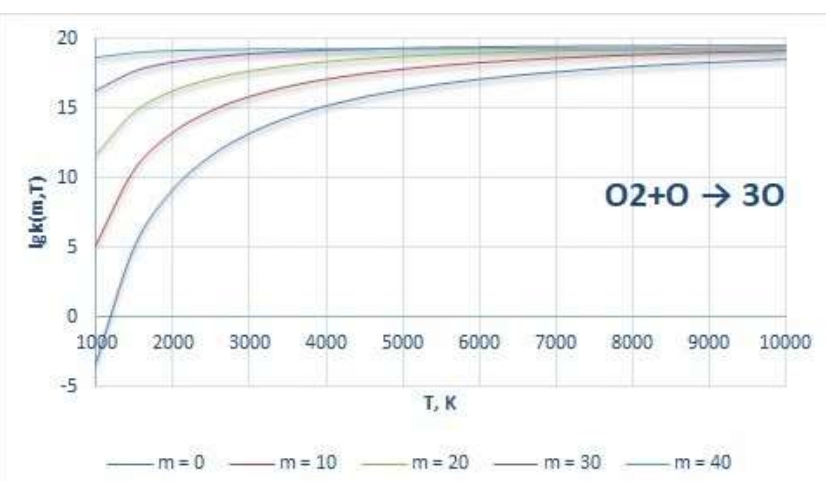

Рис. 4. Уровневая константа скорости диссоциации кислорода в зависимости от поступательной температуры

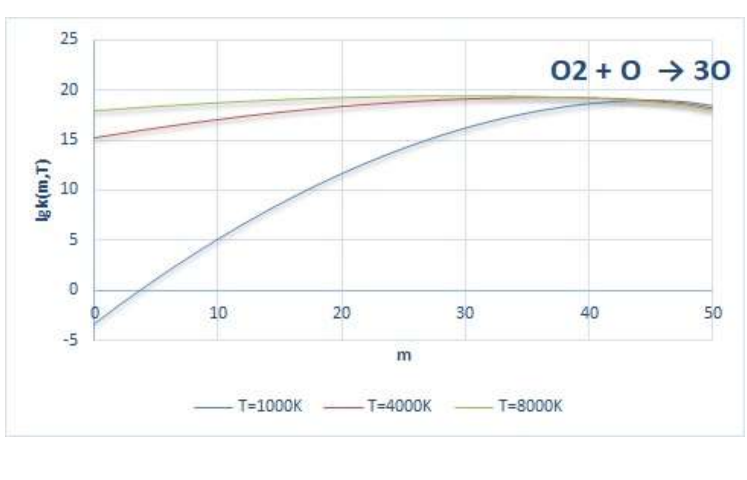

Рис. 5. Уровневая константа скорости диссоциации кислорода в зависимости от колебательного уровня

\section{ЦФ 95. Относительный уровневый фактор $G(m, m *, T)$}

- $[\mathrm{G}]=\sigma / \mathrm{p}$;

- аргумент целевой функции: $T$;

- параметр целевой функции: $m$;

- ограничения:

1. аргумента: $300 \leq T \leq D_{0} / 2$;

2. параметра: $0 \leq m \leq m_{\max }^{h}$;

- замечания:

$\mathrm{AB}(\mathrm{m})$ - ангармонический осциллятор.

\section{Расчетные формулы}

$$
G(m, T)=\frac{\exp \left(-\beta \sqrt{\Delta E_{m}}\right)}{\exp \left(-\beta \sqrt{\Delta E_{m^{*}}}\right)} \exp \left(\frac{\Delta E_{m^{*}}-\Delta E_{m}}{T}\right)\left(\frac{\Delta E_{m}}{\Delta E_{m^{*}}}\right)\left(\frac{2 T+\Delta E_{m}}{2 T+\Delta E_{m^{*}}}\right)
$$

обозначения - см. выше

\section{Характерные величины и примеры}

Для реакции диссоциации молекулы СО при столкновении с Ar был выбран температурный диапазон $T=4000 \div 14000 \mathrm{~K}$ и параметрический список $\{m\}=0,20,40,60$. Построен относительный уровневый фактор этой реакции (рис. 6 показано) на выбранных диапазонах.

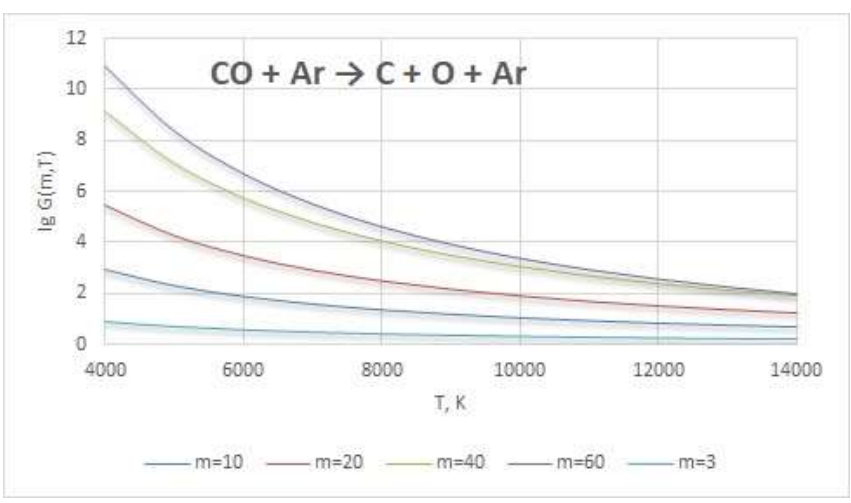

Рис. 6. Относительный уровневый фактор диссоциации $\mathrm{CO}+\mathrm{Ar} \rightarrow \mathrm{C}+\mathrm{O}+\mathrm{Ar}$ в зависимости от температуры для разных колебательных уровней 
На рис. 7 показан расчет относительного уровневого фактора на диапазоне колебательных чисел $m=0 \div 70$, для колебательной температуры в роли параметра $-\{T\}=4000,7000$, 15000 .

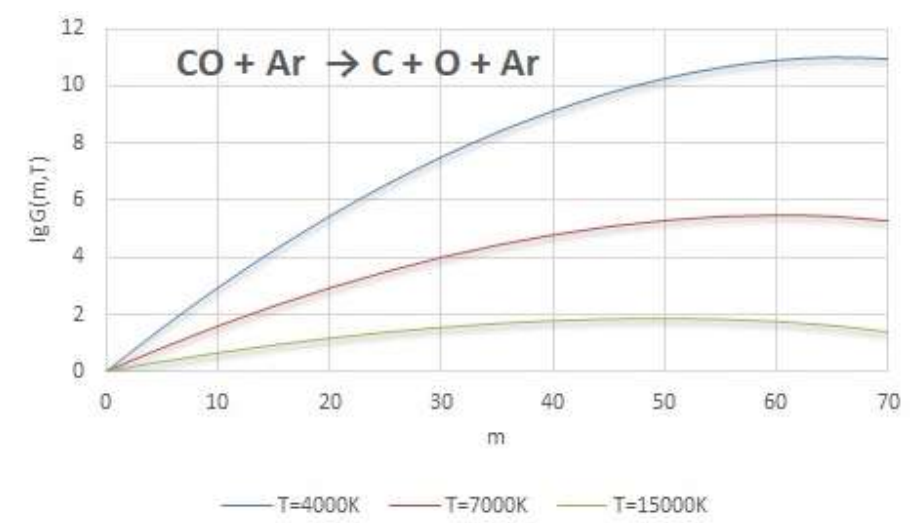

Рис. 7. Относительный уровневый фактор диссоциации $\mathrm{CO}+\mathrm{Ar} \rightarrow \mathrm{C}+\mathrm{O}+\mathrm{Ar}$ в зависимости от разных колебательных уровней для разных температур

\section{3. Сравнение уровневой адиабатической модели с другими уровневыми моделями и МКТ}

К сравнительному исследованию были привлечены следующие модели: уровневая модель Мэрроуна - Тринора и две ее модификации, одна из которых выполнена в Институте механики МГУ им. М.В. Ломоносова по результатам траекторных расчетов [24], а вторая - в Санкт-Петербургском университете А.С. Савельевым [25, 26].

Уровневая модель Мэрроуна-Тринора, впервые опубликованная в 60-х годах 20 века $[27,28]$, по-прежнему широко используется при решении задач уровневой кинетики. Она предназначена для моделирования уровневой константы скорости диссоциации двухатомных молекул в исходной уровневой кинетике, т.е. для процессов типа $\mathrm{AB}(m)+\mathrm{M} \rightarrow \mathrm{A}+\mathrm{B}+\mathrm{M}$, где исследователю известно только состояния колебательно возбужденной диссоциирующей молекулы.

В этой модели предполагается, что вероятность диссоциации экспоненциально возрастает с увеличением колебательной энергии диссоциирующей молекулы. При расчете статистических сумм принимается модель обрезанного гармонического осциллятора. Классическое выражение для константы скорости диссоциации имеет следующий вид:

$$
\begin{gathered}
k(T ; m)=k^{0}(T) \frac{Q(T)}{Q(-U)} \exp \left[E_{m}\left(\frac{1}{T}+\frac{1}{U}\right)\right], \\
Q(T)=\frac{1-\exp (-D / T)}{1-\exp (-\theta / T)}, \quad Q(-U)=\frac{1-\exp (D / U)}{1-\exp (\theta / U)}, \\
E_{m}=m \theta, \quad m=0,1,2, \ldots, m_{\max }, \quad m_{\max }=\left[\frac{D}{\theta}\right]-\text { по гармоническому осциллятору; } \\
E_{m}=m \theta \frac{1-(m+1) x_{e}}{1-2 x_{e}}, \quad x_{e}=0.25 \frac{\theta}{D}, \quad m_{\max }=2\left[\frac{D}{\theta}\right]-\text { по осциллятору Морзе }
\end{gathered}
$$

Параметр модели $U$ - условная "отрицательная температура" распределения вероятности диссоциации с разных колебательных уровней - по рекомендации автора принимается близким к величине $U=D / 6$. 
Авторами статьи по итогам сравнения модели Мэрроуна - Тринора с результатами МКТ расчетов в широком диапазоне поступательной температуры $T$ для диссоциации $\mathrm{N}_{2} \mathrm{O}_{2}$ была выполнена модификация этой модели - учтена возможная зависимость параметра $U$ от поступательной температуры. После аппроксимации результатов серии вычислительных экспериментов была предложена следующая зависимость параметра:

$$
\begin{aligned}
& U=\frac{D_{0}}{2.01} \ln (T)-15.296-\text { для диссоциации молекул } \mathrm{N}_{2}, \\
& U=D_{0}\left(0.15+\frac{T}{20000}\right)-\text { для диссоциации молекул } \mathrm{O}_{2}
\end{aligned}
$$

При анализе адекватности описания диссоциации двухатомных молекул предлагаемой уровневой адиабатической моделью также использовалось сравнение ее результатов с результатами модифицированной уровневой модели Мэрроуна - Тинора, предложенной в Санкт-Петербургском университете, далее называемой моделью Савельева. В основу этой модификации положено предположение о зависимости параметра $U$ модели Мэрроуна-Тринора от энергии конкретного колебательного уровня колебательно возбужденной диссоциирующей молекулы.

В модели Савельева параметр $U$ представлен кусочно-непрерывной функцией от энергии колебательного уровня $E_{m}$ и поступательной температуры $T$. Значения энергии колебательных уровней для реакции диссоциации $\mathrm{N}_{2}$ автор модели рекомендует брать из базы данных [29]. Однако, он же утверждает, что предложенная им модификация модели Мэрроуна-Тринора дает хорошее согласие с результатами квазиклассических траекторных расчетов и зависит от энергии колебательного уровня, а не от его номера, и позволяет использовать ее для любой модели колебательного спектра молекул.

Для сравнительных расчетов мы пользовались спектром колебательных уровней двухатомной молекулы с учетом ангармонизма

$$
E_{m}=(m+0.5) \omega_{e}-(m+0.5)(m+0.5) \omega_{e} x_{e},
$$

где $\omega_{e}$ - колебательная постоянная частицы; $\omega_{e} x_{e}-$ параметр ангармоничности.

Для $\mathrm{N}_{2}: \omega_{e}=2358.027 \mathrm{~cm}^{-1}=0.29235 \mathrm{eV}, \omega_{e} x_{e}=14.1351 \mathrm{~cm}^{-1}=0.0018 \mathrm{eV}$.

При расчете уровневых констант скорости реакции диссоциации $\mathrm{N}_{2}$ использовались данные по равновесной константе скорости [7, 30]

$$
k(T)=8.5 \times 10^{25} T^{-2.5} \exp \left(\frac{113200}{T}\right)
$$

Ниже представлено сравнение уровневой адиабатической модели (кривые результатов, полученные по ней, обозначены на графиках как TSm) с классической моделью Мэрроуна - Тринора (кривые результатов, полученные по ней, обозначены на графиках как ТМ), с моделью Савельева (обозначена как TS), с модифицированной моделью Мэрроуна - Тринора с учетом параметра $U=U(T)$ (обозначена как Tqt) и с результатом квазиклассических расчетов, которые обозначены на всех рисунках как TQ.

Поведение относительного уровневого фактора в зависимости от изменения номера колебательного уровня молекулы $\mathrm{N}_{2}$ для выбранных значений поступательной температуры $\{T\}=2000,4000,6000,8000,10000 \mathrm{~K}$ представлено на рис. 8-12. Для исследования был выбран диапазон номеров колебательных уровней от нулевого до 40 поскольку для более высоких колебательных уровней практически во всех моделях есть те или иные ограничения и расхождения. 
$G\left(m, m^{*},\{T\}\right), m^{*}=0$

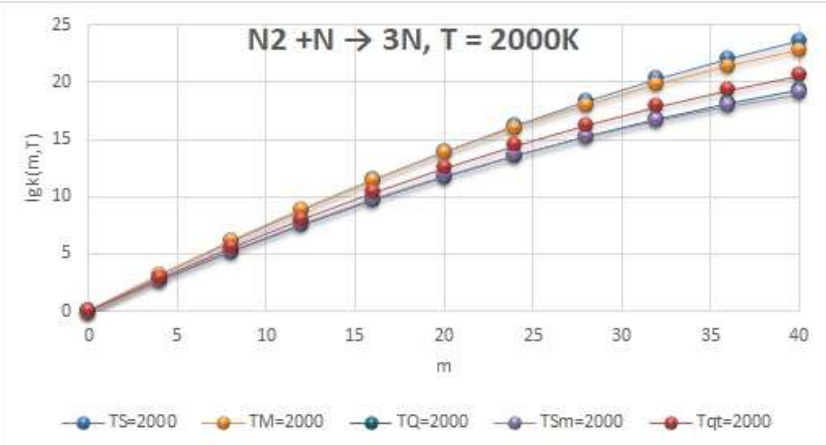

Рис. 8. Относительный уровневый фактор реакции $\mathrm{N}_{2}+\mathrm{N}=3 \mathrm{~N}$, полученный по разным моделям для среды с поступательной температурой равной $T=2000 \mathrm{~K}$

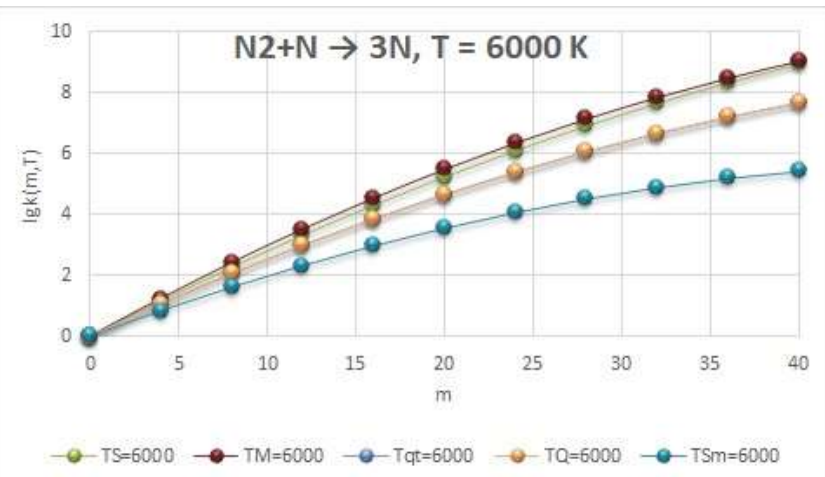

Рис. 10. Относительный уровневый фактор реакции $\mathrm{N}_{2}+\mathrm{N}=3 \mathrm{~N}$, полученный по разным моделям для среды с поступательной температурой равной $T=6000 \mathrm{~K}$

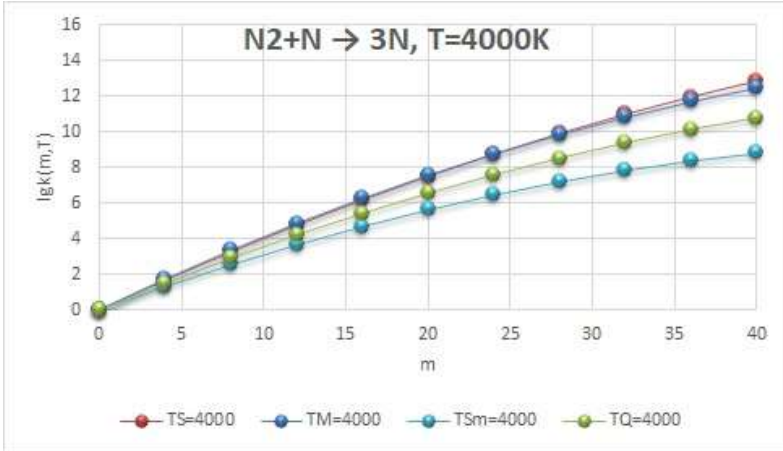

Рис. 9. Относительный уровневый фактор реакции $\mathrm{N}_{2}+\mathrm{N}=3 \mathrm{~N}$, полученный по разным моделям для среды с поступательной температурой равной $T=4000 \mathrm{~K}$

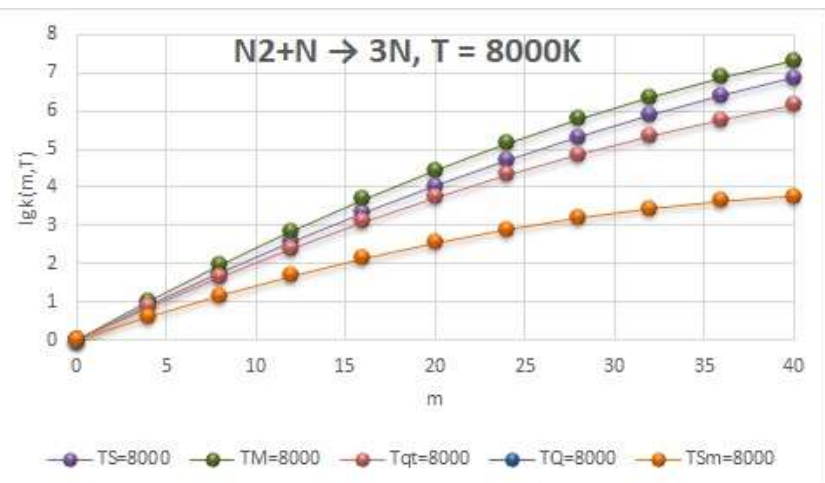

Рис. 11. Относительный уровневый фактор реакции $\mathrm{N}_{2}+\mathrm{N}=3 \mathrm{~N}$, полученный по разным моделям для среды с поступательной температурой равной $T=8000 \mathrm{~K}$

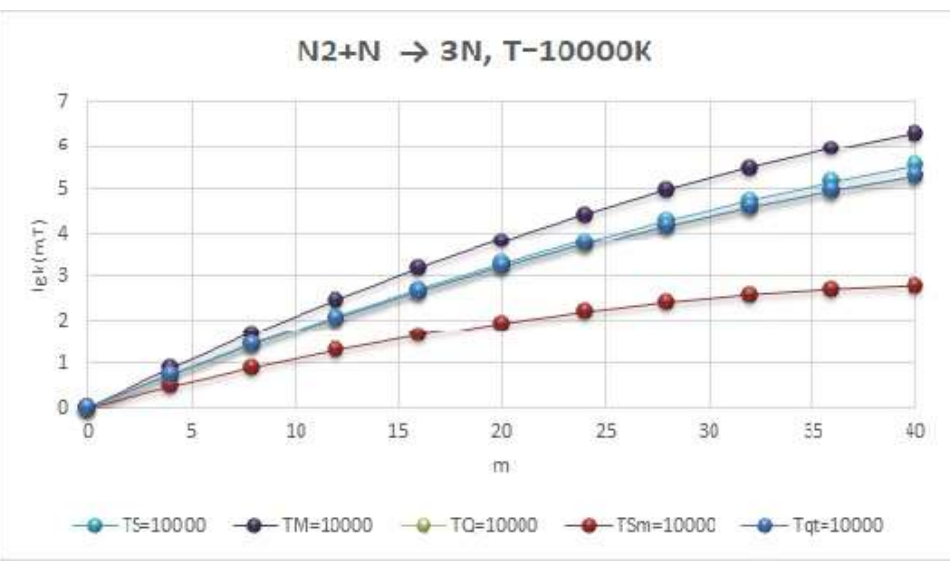

Рис. 12. Относительный уровневый фактор реакции $\mathrm{N}_{2}+\mathrm{N}=3 \mathrm{~N}$, полученный по разным моделям для среды с поступательной температурой равной $T=10000 \mathrm{~K}$

На приведенных рисунках (рис. 8-12) видно, что относительный уровневый фактор по предлагаемой адиабатической уровневой модели хорошо согласуется с данными траекторных расчетов при невысоких поступательных температурах. С повышением температуры фактор дает все более заниженные результаты. Модель Савельева дает результаты близкие результа- 
там классической модели Мэрроуна-Тринора. Однако, с повышением поступательной температуры ее результаты приближаются к данным траекторных расчетов и практически совпадают с ними при $T=10000 \mathrm{~K}$. Классическая модель Мэрроуна-Тринора не совпадает с данными МКТ ни при каких температурах, и поэтому мы рекомендуем использовать для диссоциации $\mathrm{N}_{2}$ модифицированную нами модель Мэрроуна-Тринора с параметром $U=U(T)$, а при температурах больше $8000 \mathrm{~K}$ и модель Савельева, если возникает задача включить в рассмотрение зависимость параметра $U$ от номера колебательного состояния.

$G(\{m\}, m * T), m *=0$

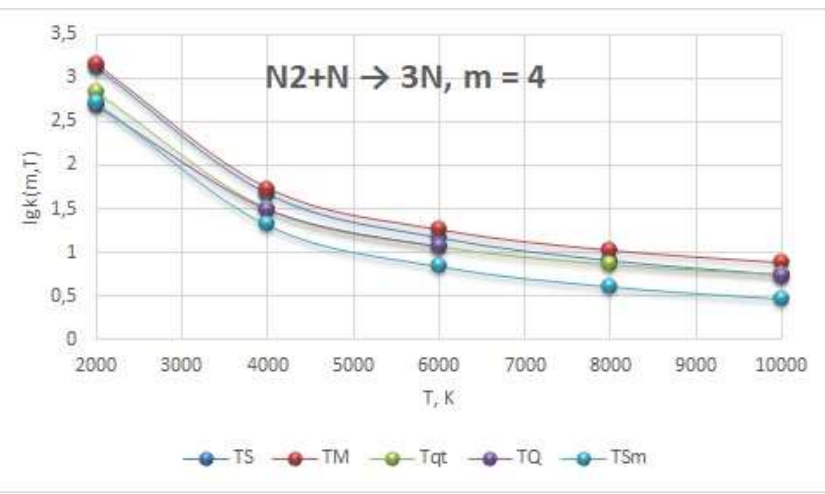

Рис. 13. Зависимость относительного уровневого фактора реакции $\mathrm{N}_{2}+\mathrm{N}=3 \mathrm{~N}$, полученная по разным моделям для колебательного уровня $m=4$

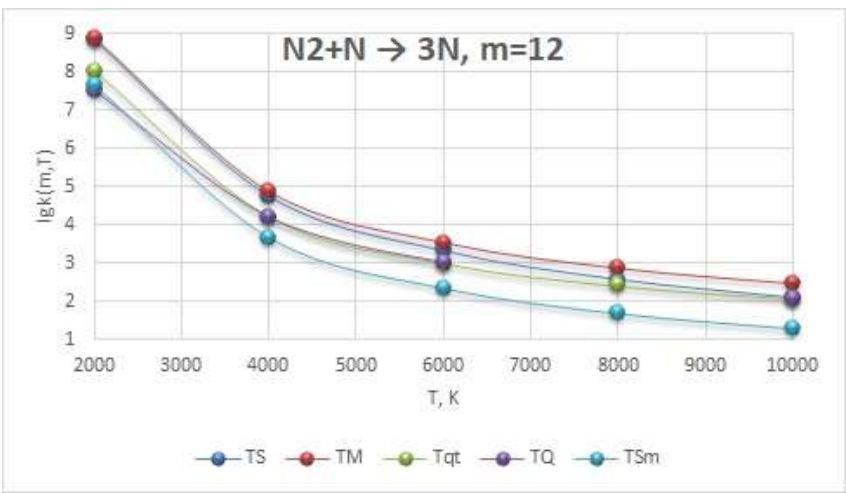

Рис. 14. Зависимость относительного уровневого фактора реакции $\mathrm{N}_{2}+\mathrm{N}=3 \mathrm{~N}$, полученная по разным моделям для колебательного уровня $m=12$

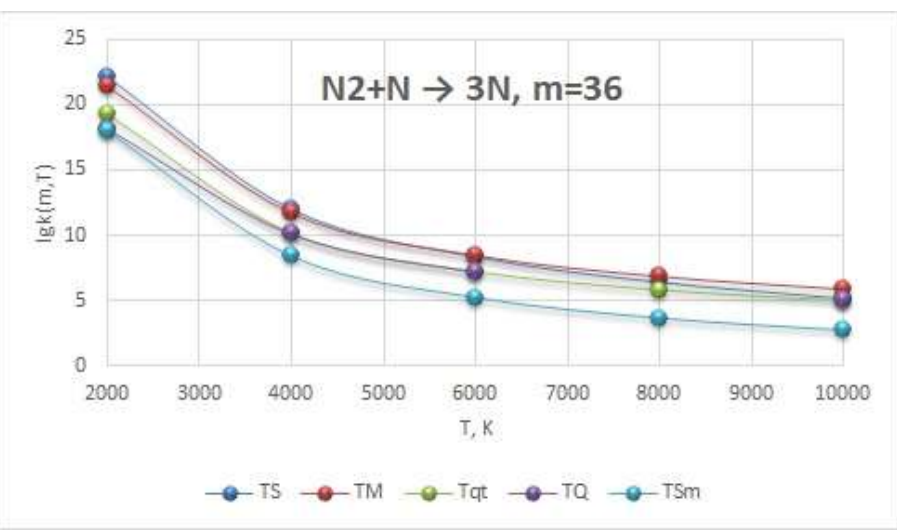

Рис. 15. Зависимость относительного уровневого фактора реакции $\mathrm{N}_{2}+\mathrm{N}=3 \mathrm{~N}$, полученная по разным моделям для колебательного уровня $m=36$

На рис. 13-15 видно, что уровневая адиабатическая модель для всех выделенных для исследования колебательных уровней дает более низкие значения относительного уровневого фактора по сравнению с другими моделями. А модель Савельева начинает давать заметные отклонения от классической модели Мэрроуна - Тринора при росте температуры, что хорошо видно при малых значениях $m$.

Уровневая константа скорости по адиабатической модели практически совпадает с данными МКТ при низких значениях поступательной температуры $T$ (рис. 16) до $T=4000 \mathrm{~K}$ включительно. Ее поведение при увеличении температуры отражено на рис. 17-20. При $T=10000$ К адиабатическая модель ведет себя подобно модели Мэрроуна-Тринора, а модель Савельева - подобно данным МКТ расчета. 

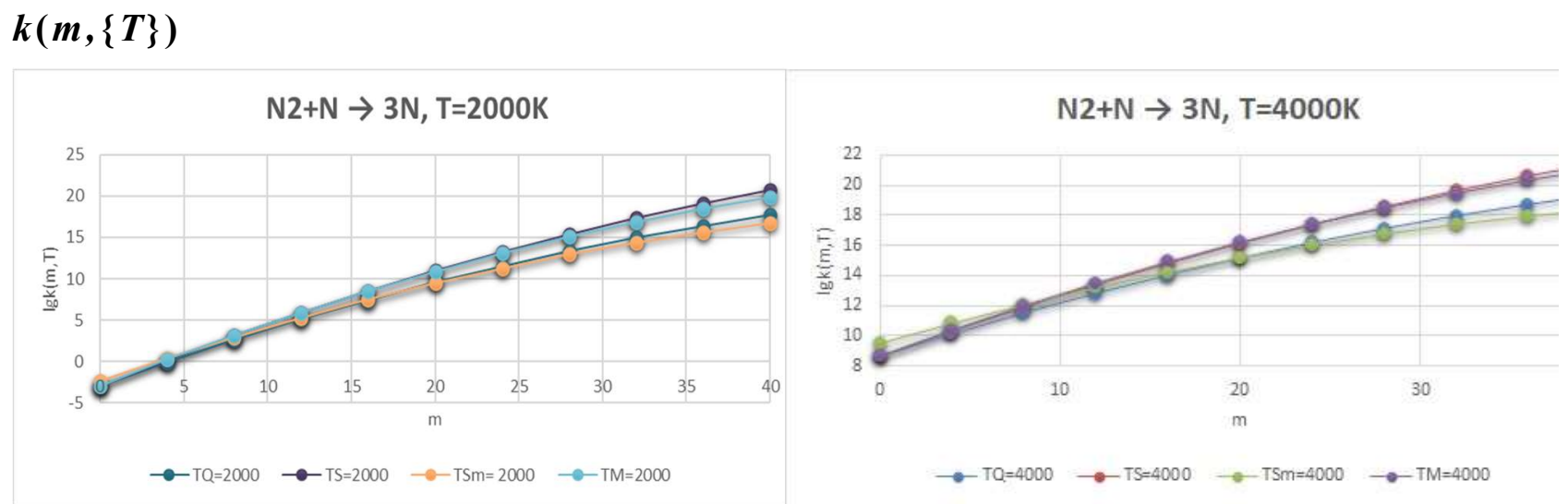

Рис. 16. Уровневая константа скорости реакции $\mathrm{N}_{2}+\mathrm{N}=3 \mathrm{~N}$, полученная по разным моделям на диапазоне колебательных уровней для среды с поступательной температурой равной $T=2000 \mathrm{~K}$

Рис. 17. Уровневая константа скорости реакции $\mathrm{N}_{2}+\mathrm{N}=3 \mathrm{~N}$, полученная по разным моделям на диапазоне колебательных уровней для среды с поступательной температурой равной $T=4000 \mathrm{~K}$
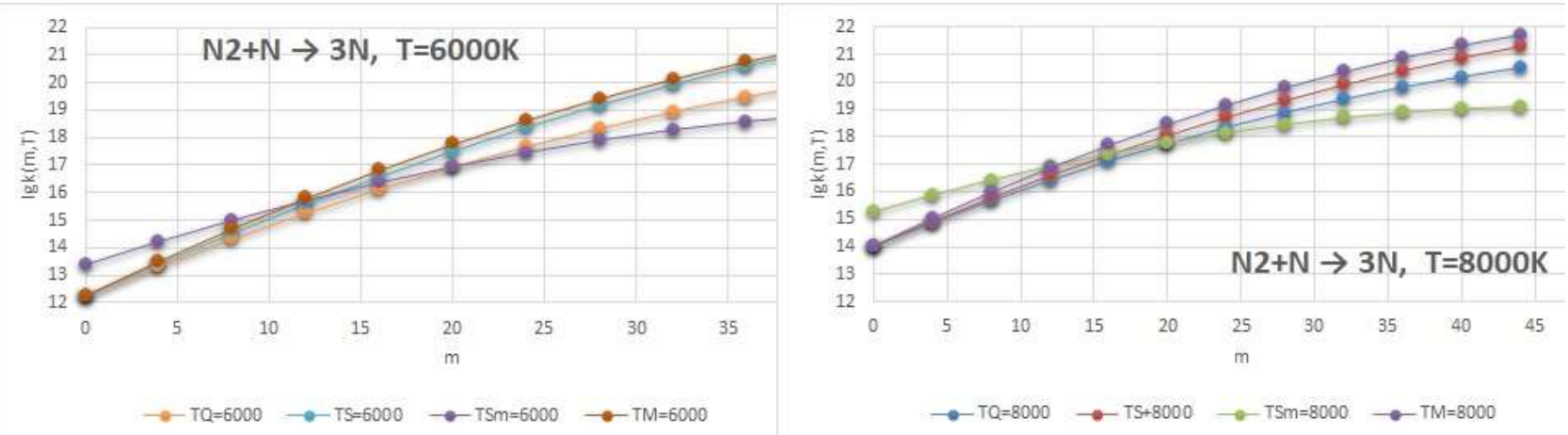

Рис. 18. Уровневая константа скорости реакции $\mathrm{N}_{2}+\mathrm{N}=3 \mathrm{~N}$, полученная по разным моделям на диапазоне колебательных уровней для среды с поступательной температурой равной $T=6000 \mathrm{~K}$

Рис. 19. Уровневая константа скорости реакции $\mathrm{N}_{2}+\mathrm{N}=3 \mathrm{~N}$, полученная по разным моделям на диапазоне колебательных уровней для среды с поступательной температурой равной $T=8000 \mathrm{~K}$

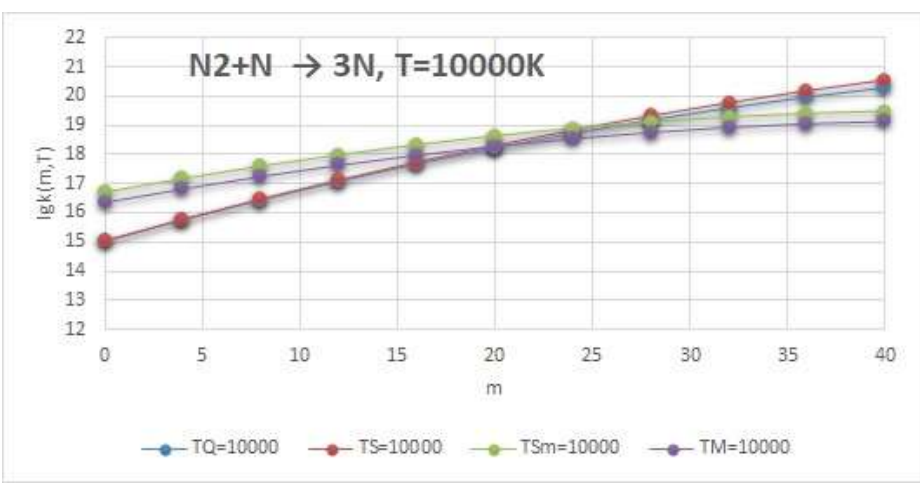

Рис. 20. Уровневая константа скорости реакции $\mathrm{N}_{2}+\mathrm{N}=3 \mathrm{~N}$, полученная по разным моделям на диапазоне колебательных уровней для среды с поступательной температурой равной $T=10000 \mathrm{~K}$

Если рассматривать значения уровневой константы скорости для разных колебательных уровней молекулы азота, то видно, что для низких $m$ есть небольшое отличие значений константы скорости по разным моделям (рис. 21), для средних $m$ - практически все модели дают одинаковые результаты (рис. 22), а для высоких колебательных уровней адиабатическая модель лежит ниже данных по МКТ (рис. 23). 
$k(\{m\}, T)$

Для $T=2000 \div 10000 \mathrm{~K} \mathrm{с} \mathrm{шагом} 2000 \mathrm{~K}$ для $m=\{4,12,36\}$
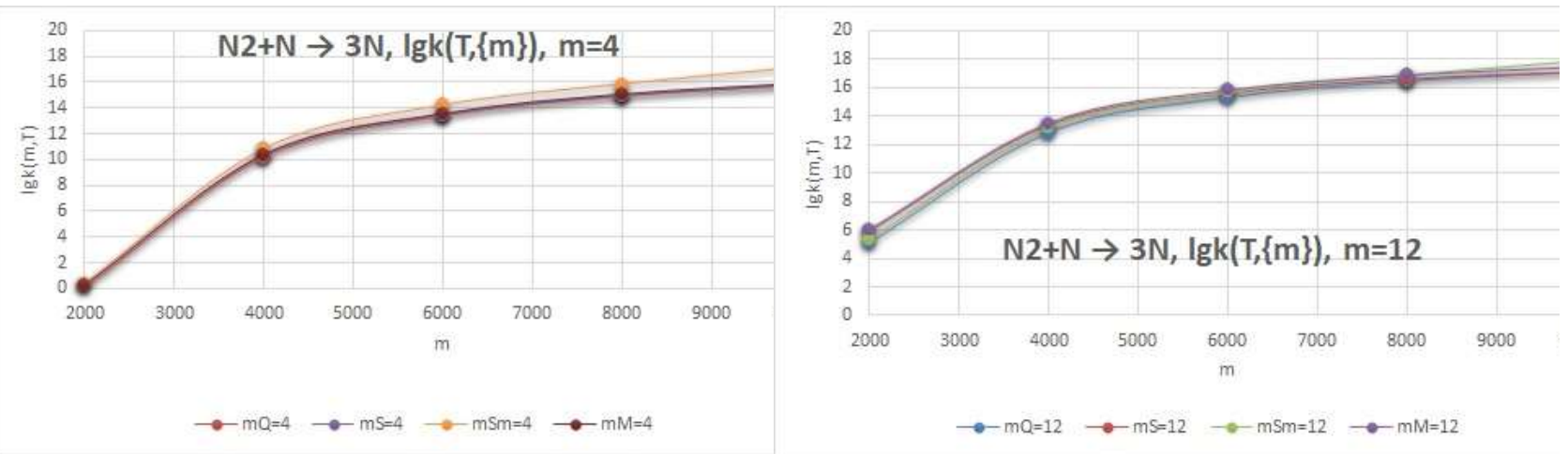

Рис. 21. Зависимость уровневой константы скорости реакции $\mathrm{N}_{2}+\mathrm{N}=3 \mathrm{~N}$, полученная по разным моделям на диапазоне температуры для колебательного уровня $m=4$

Рис. 22, Зависимость уровневой константы скорости реакции $\mathrm{N}_{2}+\mathrm{N}=3 \mathrm{~N}$, полученная по разным моделям на диапазоне температуры для колебательного уровня $m=12$

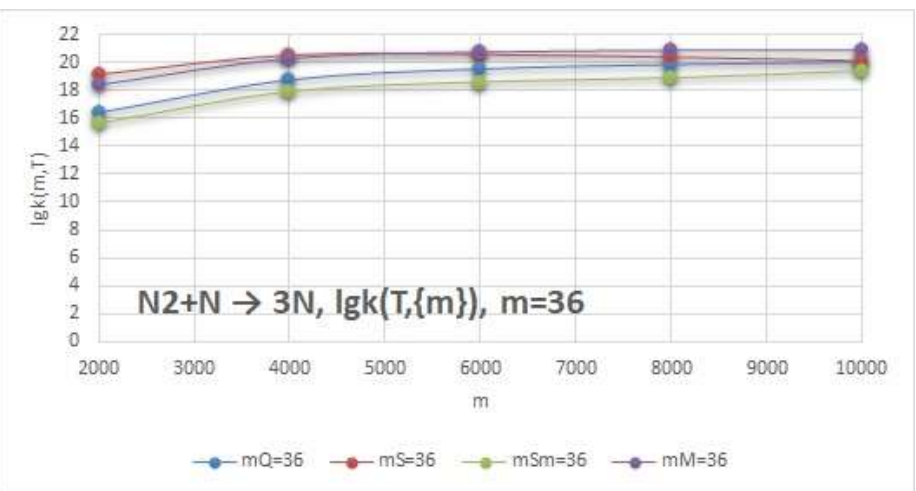

Рис. 23. Зависимость уровневой константы скорости реакции $\mathrm{N}_{2}+\mathrm{N}=3 \mathrm{~N}$, полученная по разным моделям на диапазоне температуры для колебательного уровня $m=36$

Можно сделать вывод, что уровневая адиабатическая модель может быть рекомендована для задач, в которых рассматриваются невысокие температуры. Она требует знания только фундаментальных характеристик диссоциирующей молекулы, достаточно проста в вычислениях и адекватно описывает диссоциацию различных молекул.

\section{4. Заключение}

Предложена новая уровневая модель диссоциации двухатомных молекул. Приведены характерные величины модели на примере диссоциации молекул $\mathrm{O}_{2}, \mathrm{~N}_{2}$ и СО. Новая модель достаточно адекватно описывает процесс диссоциации особенно при невысоких поступательных температурах, то, поскольку для ее реализации требуется знания только справочных данных о двухатомной молекуле и ее партнере по столкновению, то ее можно смело рекомендовать для оценки процесса диссоциации широкого набора двухатомных молекул.

Проведено сравнительное исследование трех уровневых моделей диссоциации - классической модели Мэрроуна - Тринора, модифицированной модели Мэрроуна - Тринора, модели Савельева - и результатов МКТ расчета на примере диссоциации молекулы $\mathrm{N}_{2}$ в широком диапазоне поступательной температуры от $2000 \mathrm{~K}$ до $10000 \mathrm{~K}$ и для диапазона колебательных чисел - от 0 до 40. 


\section{Благодарности и ссылки на гранты}

Работа выполнена в соответствии с планом научных исследований НИИ механики МГУ им. М.В. Ломоносова при частичной финансовой поддержке РФФИ в рамках научного проекта № 18-01-00741А.

\section{Литература}

1. Физико-химические процессы в газовой динамике (под ред. Г. Г. Черного, С. А. Лосева). М.: Издво Моск. ун-та. 1995. 350 с.

2. Нагнибеда Е.А., Кустова Е.В. Кинетическая теория процессов переноса и релаксации в потоках неравновесных реагирующих газов. СПб.: Изд-во С.-Петерб. ун-та, 2003. 272 с.

3. Кондратьев В.Н., Никитин Е.Е. Кинетика и механизм газофазных реакций. М.: Наука, 1974. 558 с.

4. Ступоченко Е.В., Лосев С.А., Осипов А.И. Релаксационные процессы в ударных волнах. М.: Наука, 1965. 484 c.

5. Кривоносова О.Э., Лосев С.А., Наливайко В.П., Мукосеев Ю. К., Шаталов О.П. Рекомендуемые данные о константах скорости химических реакций между молекулами, состоящими из атомов N и О// Химия плазмы. Энергоатомиздат. Москва, 1987. Т.14. С. 3-31.

6. NIST Chemical Kinetics Database [Электронный ресурс] https://kinetics.nist.gov/kinetics/welcome.jsp

7. Ковач Э.А., Лосев С.А., Сергиевская А.Л., Храпак Н.А. Каталог моделей физико-химических процессов 3. Термически равновесные и неравновесные химические реакции//Физико-химическая кинетика в газовой динамике. 2010. Т. 10. http://chemphys.edu.ru/issues/2010-10/articles/333/

8. Armenise I., Esposito F. Dissociation-recombination models in hypersonic boundary layer $\mathrm{O}_{2} / \mathrm{O}$ flows // Chemical Physics. 398. 2012. Pp. 104-110.

9. Armenise I., Esposito F., Capitta G., Capitelli M. O-O $\mathrm{O}_{2}$ state-to-state vibrational relaxation and dissociation rates based on quasiclassical calculations // Chemical Physics. 351. 2008. Pp. 91-98.

10. Bender J.D., Valentini P., Nompelis I., Paukku Y., Varga Z., Truhlar D. G., Schwartzentruber T., Candler G.V. An improved potential energy surface and multi-temperature quasiclassical trajectory calculations of $\mathrm{N}_{2}+\mathrm{N}_{2}$ dissociation reactions// J. Chem. Phys. 2015. Vol. 143. P. 054304 (23pp). doi:10.1063/1.4927571

11. Pogosbekian M.J., Sergievskaia A.L., Losev S.A. Verification of theoretical models of chemical exchange reactions on the basis of quasiclassical trajectory calculations // Chemical Physics. 2006. Vol. 328, No. 1-3. Pp. 371-378.

12. Погосбекян М.Ю., Лосев С.А. Исследование реакции $\mathrm{CO}+\mathrm{N}->\mathrm{CN}+\mathrm{O}$ методом квазиклассических траекторий с использованием вычислительного комплекса "MD TRAJECTORY" // Химическая физика. 2003. Т. 22, № 6. С. 38-46.

13. Grover M.S., Schwartzentruber T.E., Internal Energy Relaxation and Dissociation in Molecular Oxygen Using Direct Molecular Simulation, $47^{\text {th }}$ AIAA Thermophysical Conference. AIAA Paper 2017-3488. 2017. doi:10.2514/6.2017-3488

14. Andrienko D., Boyd I.D., Investigation of Oxygen Vibrational Relaxation by Quasy-Classical Trajectory Method, Chemical Physics. Vol.459. No. 28. 2015. Pp. 1-13. doi:10.1016/j.chemphys.2015.07.023

15. Yakunchikov A., Kosyanchuk V., Kroupnov A., Pogosbekian M., Bryukhanov I., Iuldasheva A. Potential energy surface of interaction of two diatomic molecules for air flows simulation at intermediate temperatures. Chemical Physics. 536:110850. 2020. doi: 10.1016/j.chemphys.2020.110850

16. Савельев А.С. Модели коэффициентов скорости химических реакций для задач неравновесной аэродинамики. Диссер на соискание степени к.ф-м.н., - Санкт-Петербург. 2018. 118 с. 
17. Погосбекян М. Ю., Сергиевская А. Л., Крупнов А. А. Сравнительное моделирование процесса диссоциации молекул $\mathrm{N}_{2}$ в термически неравновесных условиях // Труды МАИ. 2018. № 102. C. $1-30$.

18. Смехов Г.Д., Яловик М.С. Диссоциация молекул азота в колебательно неравновесном газе // Химическая физика. 1996. Т.15. N 4. С. 17-35.

19. Смехов Г. Д., Жлуктов С. В. Константа скорости диссоциации двухатомных молекул в адиабатической модели // Химическая физика. 1992. Т. 11, № 9. С. 1171-1179.

20. Смехов Г. Д. Применение адиабатического принципа для вычисления константы скорости диссоциации двухатомных молекул//Неравновесные течения газа и оптимальные формы тел в гиперзвуковом потоке. Изд. Московского университета. Москва, 1982. С. 30-38.

21. Смехов Г. Д., Лосев С. А. О роли колебательно-вращательного возбуждения в процессе диссоциации двухатомных молекул // Теоретическая и экспериментальная химия. 1979. Т. 15. № 5. C. $492-497$.

22. Sergievskaya A., Pogosbekian M. The study of oxygen dissociation models based on quasi-classical calculations in thermal nonequilibrium conditions // Proceedings of the International Conference on Numerical Analysis and Applied Mathematics 2016. Vol. 1863. United States, 2016. Pp. 110003-1110003-4. doi: 10.1063/1.4992288

23. Погосбекян М. Ю., Сергиевская А. Л. Моделирование реакции диссоциации кислорода в термически неравновесных условиях: модели, траекторные расчеты, эксперимент // Химическая физика. 2018. Т. 37, № 4. С. 20-31. doi:10.7868/S0207401X18040039

24. Погосбекян М.Ю., Сергиевская А.Л. Моделирование динамики молекулярных реакций и сравнительный анализ с теоретическими моделями применительно к термически неравновесным условиям//Физико-химическая кинетика в газовой динамике. 2014. T. 15, Вып. 3. http://chemphys.edu.ru/issues/2014-15-3/articles/227/

25. Кустова Е.В., Савельев А.С., Лукашева А.А. Уточнение поуровневых моделей химической кинетики с помощью данных траекторных расчетов//Физико-химическая кинетика в газовой динамике. 2018. Т. 19, вып. 3. http://chemphys.edu.ru/issues/2018-19-3/articles/767/. doi:10.33257/PhChGD.19.3.767

26. Савельев А.С., Кустова Е.В. Пределы применимости модели Тринора-Маррона для поуровневых коэффициентов скорости диссоциации $\mathrm{N}_{2}$ и $\mathrm{O}_{2} / /$ Вестник СПбГУ. Сер. 1. 2015. Е. 2 (60), № 2. C. 266-277.

27. Treanor C.E., Marrone P.V. Effect of dissociation on the rate of vibrational relaxation // The Physics of Fluids. 1962. Vol. 5. No. 9. Pp. 1022-1026.

28. Marrone P.V., Treanor C.E. Chemical relaxation with preferential dissociation from excited vibrational levels // The Physics of Fluids. 1963. Vol. 6, No. 9. Pp. 1215-1221.

29. Planetary Entry Integrated models. [Электронный ресурс]: http://phys4entrydb.ba.imip.cnr.it/Phys4EntryDB/

30. Ковач Э.А., Кривоносова О.Э., Лосев С.А., Мукосеев Ю.К., Наливайко В.П., Христенко А.В., Шаталов О.П. База рекомендуемых данных первой версии макета системы АВОГАДРО. // Отчет НИИ Механики № 3312. 1986. 41 с.

\section{References}

1. Fiziko-himicheskie processy v gazovoj dinamike (Physical and chemical processes in gas dynamics) (Ed. G. G. Chernogo, S. A. Loseva), M.: Izd-vo Mosk. un-ta, 1995, 350 p.

2. Nagnibeda, E. A., Kustova, E. V., Kineticheskaja teorija processov perenosa i relaksacii v potokah neravnovesnyh reagirujushih gazov (Kinetic Theory of Transfer and Relaxation Processes in Flows of Nonequilibrium Reacting Gases), SPb.: Izd-vo S.-Peterb. un-ta, 2003, 272 p. 
3. Kondrat'ev, V. N., Nikitin, E. E., Kinetika i mehanizm gazofaznyh reakcij (Kinetics and mechanism of gasphase reactions), M.: Nauka, 1974, 558 p.

4. Stupochenko, E. V., Losev, S. A., Osipov, A. I., Relaksacionnye processy v udarnyh volnah (Relaxation processes in shock waves), M.: Nauka, 1965, 484 p.

5. Krivonosova, O. Je., Losev, S. A., Nalivajko, V. P., Mukoseev, Ju. K., Shatalov, O. P., "Rekomenduemye dannye o konstantah skorosti himicheskih reakcij mezhdu molekulami, sostojashhimi iz atomov N i O," Himija plazmy, Jenergoatomizdat, Moskva, Vol. 14, 1987, pp. 3-31.

6. NIST Chemical Kinetics Database [Jelektronnyj resurs] https://kinetics.nist.gov/kinetics/welcome.jsp

7. Kovach, Je. A., Losev, S. A., Sergievskaja, A. L., Hrapak, N. A., "Katalog modelej fiziko-himicheskih processov 3. Termicheski ravnovesnye i neravnovesnye himicheskie reakcii," Fiziko-himicheskaja kinetika v gazovoj dinamike (Physical-Chemical Kinetics in Gas Dynamics), Vol. 10, 2010. http://chemphys.edu.ru/issues/2010-10/articles/333/

8. Armenise I., Esposito F. Dissociation-recombination models in hypersonic boundary layer $\mathrm{O}_{2} / \mathrm{O}$ flows // Chemical Physics. 398. 2012. Pp. 104-110.

9. Armenise, I., Esposito, F., Capitta, G., Capitelli, M., "O-O $\mathrm{O}_{2}$ state-to-state vibrational relaxation and dissociation rates based on quasiclassical calculations," Chemical Physics, Vol. 351, 2008, pp. 91-98.

10. Bender, J. D., Valentini, P., Nompelis, I., Paukku, Y., Varga, Z., Truhlar, D. G., Schwartzentruber, T., Candler, G. V., "An improved potential energy surface and multi-temperature quasiclassical trajectory calculations of $\mathrm{N}_{2}+\mathrm{N}_{2}$ dissociation reactions," J. Chem. Phys., Vol. 143, 2015, P. 054304 (23pp). doi:10.1063/1.4927571

11. Pogosbekian, M. J., Sergievskaia, A. L., Losev, S. A., "Verification of theoretical models of chemical exchange reactions on the basis of quasiclassical trajectory calculations," Chemical Physics, Vol. 328, No. 1-3, 2006, pp. 371-378.

12. Pogosbekjan, M. Ju., Losev, S. A., "Issledovanie reakcii $\mathrm{CO}+\mathrm{N}->\mathrm{CN}+\mathrm{O}$ metodom kvaziklassicheskih traektorij s ispol'zovaniem vychislitel'nogo kompleksa "MD TRAJECTORY"," Himicheskaja fizika, Vol. 22, No. 6, 2003, pp. 38-46.

13. Grover, M. S., Schwartzentruber, T. E., "Internal Energy Relaxation and Dissociation in Molecular Oxygen Using Direct Molecular Simulation," 47 ${ }^{\text {th }}$ AIAA Thermophysical Conference, AIAA Paper 20173488, 2017. doi:10.2514/6.2017-3488

14. Andrienko, D., Boyd, I. D., "Investigation of Oxygen Vibrational Relaxation by Quasy-Classical Trajectory Method," Chemical Physics, Vol. 459, No. 28, 2015, pp. 1-13. doi:10.1016/j.chemphys.2015.07.023

15. Yakunchikov, A., Kosyanchuk, V., Kroupnov, A., Pogosbekian, M., Bryukhanov, I., Iuldasheva, A., "Potential energy surface of interaction of two diatomic molecules for air flows simulation at intermediate temperatures," Chemical Physics, Vol. 536:110850, 2020. doi: 10.1016/j.chemphys.2020.110850

16. Savel'ev, A. S., Modeli kojefficientov skorosti himicheskih reakcij dlja zadach neravnovesnoj ajerodinamiki (Models of chemical reaction rate coefficients for nonequilibrium aerodynamics problems), Disser na soiskanie stepeni k.f-m.n., - Sankt-Peterbur. 2018. 118 p.

17. Pogosbekjan, M. Ju., Sergievskaja, A. L., Krupnov, A. A., "Sravnitel'noe modelirovanie processa dissociacii molekul $\mathrm{N}_{2}$ v termicheski neravnovesnyh uslovijah," Trudy MAI, No. 102, 2018, pp. 1-30.

18. Smehov, G. D., Jalovik, M. S., "Dissociacija molekul azota v kolebatel'no neravnovesnom gaze," Himicheskaja fizika, Vol. 15, No. 4, 1996, pp. 17-35.

19. Smehov, G. D., Zhluktov, S. V., "Konstanta skorosti dissociacii dvuhatomnyh molekul v adiabaticheskoj modeli," Himicheskaja fizika, Vol. 11, No. 9, 1992, pp. 1171-1179.

20. Smehov, G. D., "Primenenie adiabaticheskogo principa dlja vychislenija konstanty skorosti dissociacii dvuhatomnyh molekul," Neravnovesnye techenija gaza i optimal'nye formy tel v giperzvukovom potoke, Izd. Moskovskogo universiteta, Moskva, 1982, pp. 30-38. 
21. Smehov, G. D., Losev, S. A., "O roli kolebatel'no-vrashhatel'nogo vozbuzhdenija v processe dissociacii dvuhatomnyh molekul," Teoreticheskaja i jeksperimental'naja himija, Vol. 15, No. 5, 1979, pp. 492-497.

22. Sergievskaya, A., Pogosbekian, M., "The study of oxygen dissociation models based on quasi-classical calculations in thermal nonequilibrium conditions," Proceedings of the International Conference on Numerical Analysis and Applied Mathematics, Vol. 1863. United States, 2016, pp. 110003-1-1100034. doi: 10.1063/1.4992288

23. Pogosbekjan, M. Ju., Sergievskaja, A. L., "Modelirovanie reakcii dissociacii kisloroda v termicheski neravnovesnyh uslovijah: modeli, traektornye raschety, jeksperiment," Himicheskaja fizika, Vol. 37, No. 4, 2018, pp. 20-31. doi:10.7868/S0207401X18040039

24. Pogosbekjan, M. Ju., Sergievskaja, A. L., Modelirovanie dinamiki molekuljarnyh reakcij i sravnitel'nyj analiz s teoreticheskimi modeljami primenitel'no k termicheski neravnovesnym uslovijam," Fizikohimicheskaja kinetika v gazovoj dinamike (Physical-Chemical Kinetics in Gas Dynamics), Vol. 15, No. 3, 2014. http://chemphys.edu.ru/issues/2014-15-3/articles/227/

25. Kustova, E. V., Savel'ev, A. S., Lukasheva, A. A., "Utochnenie pourovnevyh modelej himicheskoj kinetiki s pomoshh'ju dannyh traektornyh raschetov," Fiziko-himicheskaja kinetika v gazovoj dinamike (Physical-Chemical Kinetics in Gas Dynamics), Vol. 19, No. 3, 2018. http://chemphys.edu.ru/issues/2018-19-3/articles/767/. doi:10.33257/PhChGD.19.3.767

26. Savel'ev, A. S., Kustova, E. V., "Predely primenimosti modeli Trinora-Marrona dlja pourovnevyh kojefficientov skorosti dissociacii $\mathrm{N}_{2}$ i O $\mathrm{O}_{2}$,"Vestnik $S P b G U$, Ser. 1, E. 2 (60), No. 2, 2015. pp. 266-277.

27. Treanor, C. E., Marrone, P. V., "Effect of dissociation on the rate of vibrational relaxation," The Physics of Fluids, Vol. 5. No. 9, 1962, pp. 1022-1026.

28. Marrone, P. V., Treanor, C. E., "Chemical relaxation with preferential dissociation from excited vibrational levels," The Physics of Fluids, Vol. 6, No. 9, 1963, pp. 1215-1221.

29. Planetary Entry Integrated models. [Jelektronnyj resurs]: http://phys4entrydb.ba.imip.cnr.it/Phys4EntryDB/

30. Kovach, Je. A., Krivonosova, O. Je., Losev, S. A., Mukoseev, Ju. K., Nalivajko, V. P., Hristenko, A. V., Shatalov, O. P., "Baza rekomenduemyh dannyh pervoj versii maketa sistemy AVOGADRO," Otchet NII Mehaniki MGU, No. 3312, 1986, 41 p.

Статья поступила в редакцию 27 ноября 2020 г. 